\title{
Corticospinal Beta-Band Synchronization Entails Rhythmic Gain Modulation
}

\author{
Gijs van Elswijk, ${ }^{1,2,3}$ Femke Maij, ${ }^{1}$ Jan-Mathijs Schoffelen, ${ }^{2}$ Sebastiaan Overeem, ${ }^{1}$ Dick F. Stegeman, ${ }^{1}$ and Pascal Fries ${ }^{2,4}$ \\ ${ }^{1}$ Department of Clinical Neurophysiology, Radboud University Nijmegen Medical Centre and ${ }^{2}$ Centre for Cognitive Neuroimaging, Radboud University \\ Nijmegen, Donders Institute for Brain, Cognition, and Behaviour, 6525 EN Nijmegen, The Netherlands, ${ }^{3}$ Philips Research Europe, 5656 AE Eindhoven, The \\ Netherlands, and ${ }^{4}$ Ernst Strüngmann Institute in Cooperation with Max Planck Society, 60528 Frankfurt, Germany
}

\begin{abstract}
Rhythmic synchronization of neurons in the beta or gamma band occurs almost ubiquitously, and this synchronization has been linked to numerous nervous system functions. Many respective studies make the implicit assumption that neuronal synchronization affects neuronal interactions. Indeed, when neurons synchronize, their output spikes reach postsynaptic neurons together, trigger coincidence detection mechanisms, and therefore have an enhanced impact. There is ample experimental evidence demonstrating this consequence of neuronal synchronization, but beyond this, beta/gamma-band synchronization within a group of neurons might also modulate the impact of synaptic input to that synchronized group. This would constitute a separate mechanism through which synchronization affects neuronal interactions, but direct in vivo evidence for this putative mechanism is lacking. Here, we demonstrate that synchronized beta-band activity of a neuronal group modulates the efficacy of synaptic input to that group in-phase with the beta rhythm. This response modulation was not an addition of rhythmic activity onto the average response but a rhythmic modulation of multiplicative input gain. Our results demonstrate that beta-rhythmic activity of a neuronal target group multiplexes input gain along the rhythm cycle. The actual gain of an input then depends on the precision and the phase of its rhythmic synchronization to this target, providing one mechanistic explanation for why synchronization modulates interactions.
\end{abstract}

\section{Introduction}

Activated neuronal groups typically engage in rhythmic synchronization in the beta-frequency $(12-30 \mathrm{~Hz})$ and/or gammafrequency $(30-100 \mathrm{~Hz})$ band, and this has been implicated in numerous nervous system functions (Singer and Gray, 1995; Schnitzler and Gross, 2005). Because of their putative functional importance, the mechanisms behind neuronal beta- and gammaband synchronization have been studied extensively (Kopell et al., 2000; Whittington et al., 2000; Csicsvari et al., 2003; Hasenstaub et al., 2005). However, although much is known about mechanisms and specific functions, we need to obtain a better understanding of the immediate consequences of synchronization, because this will allow us to understand how they can subserve function (Fries, 2005; Fries et al., 2007; Börgers and Kopell, 2008).

Many cognitive functions, such as selective attention, require the dynamic modulation of neuronal interaction strength, i.e., the modulation of the gain of neuronal connections (Reynolds et al., 1999; Salinas and Thier, 2000). We have proposed recently that neuronal interaction strength is determined mechanistically

\footnotetext{
Received June 14, 2009; revised Nov. 1, 2009; accepted Feb. 18, 2010.

This research was supported by grants from the Netherlands Organization for Scientific Research (S.O., D.F.S.

P.F.), the European Science Foundation European Young Investigator Award Program (P.F.), and the Ernst Strüngmann Institute. We thank C. van der Reijden for technical assistance and R. Oostenveld for help during data analysis. The authors declare no competing financial interests.

This article is freely available online through the J Neurosci Open Choice option.

Correspondence should be addressed to P. Fries, Ernst Strüngmann Institute in Cooperation with Max Planck

Society, Deutschordenstraße 46, 60528 Frankfurt, Germany. E-mail: pascal.fries@esi-frankfurt.de.

DOI:10.1523/JNEUROSCI.2794-09.2010

Copyright $\odot 2010$ the authors $\quad 0270-6474 / 10 / 304481-08 \$ 15.00 / 0$
}

by neuronal beta/gamma-band synchronization (Fries, 2005). Within a given neuronal group, beta and gamma rhythms entail rhythmic, synchronized inhibition (Börgers et al., 2005). This rhythmic inhibition might result in rhythmic changes in that group's susceptibility to input, i.e., its input gain. Several testable predictions follow. (1) The response to a single short-lasting input depends on the phase of the beta/gamma rhythm of the target at which the input arrives. (2) The response to beta/gamma rhythmic input correspondingly depends on the average phase and the precision at which the input is synchronized to the rhythm of the target. (3) For two mutually interacting neuronal groups, their interaction strength depends on the phase and precision of their beta/gamma rhythmic synchronization.

We have recently confirmed prediction 3 by demonstrating correlations between spontaneous variabilities in synchronization and interaction strength (Womelsdorf et al., 2007). Here, we directly test the more fundamental prediction 1 , that the response to a single short-lasting input depends on the phase of the rhythm of the target at which the input arrives. The ideal test of this prediction uses a physiological beta or gamma rhythm and produces a synaptic test input at experimenter-defined times. We therefore turned to the human motor system. When the motor system maintains isometric contractions, there is beta-band synchronization between involved neuronal groups of the motor cortex and the spinal cord (Murthy and Fetz, 1996; Schoffelen et al., 2005, 2008). A time-delayed copy of the beta rhythm of the spinal cord can be recorded as the electromyogram (EMG) and a synaptic test input to the spinal cord can be generated through transcranial magnetic stimulation (TMS) of the motor cortex. 
Although TMS over motor cortex generates a well synchronized synaptic input volley to the spinal cord, it has no perturbing direct electromagnetic effects on the spinal cord. Thus, the betaband synchronization of the human motor system in combination with TMS is the ideal test case for our hypothesis.

\section{Materials and Methods}

Subjects. Thirteen healthy volunteers participated in the experiment (five females; age range, 23-31 years). All subjects had normal or correctedto-normal visual acuity; 10 were right-handed, and the other three were left-handed [mean \pm SD Oldfield (1971) handedness scores of $89 \pm 19$ and $-88 \pm 19$, respectively]. None of the subjects had a history of neurological illness or neurosurgery, or any metal or electronic implants. The protocol was approved by the local ethics committee, and all subjects gave written informed consent before the experiment.

Behavioral task. Subjects were seated in front of a computer screen. The left hand rested on the left thigh. The right hand rested, palm down, on a wooden plate placed on the right thigh. On the plate, there were two parallel wooden beams that were adjusted such that the digits $2-5$ fitted snugly between them. We measured the EMG (for details, see below) from the first dorsal interosseus (FDI) muscle (musculus interosseus dorsalis primus). Before the task, subjects were asked to perform maximal voluntary contractions (MVCs) twice with a 1 min pause between. The average EMG amplitude of these two attempts was defined as the EMG amplitude during MVCs. During the task, subjects were required to maintain an isometric abduction of their right index finger to produce an EMG amplitude of $15 \%$ of the value during MVCs. Continuous EMG amplitude feedback was provided via a cursor on the computer screen, and subjects were instructed to keep the cursor as steady as possible on a horizontal line that indicated the required amplitude. The color of the cursor indicated whether the subjects should rest (red cursor) or perform the task (green cursor). Subjects were required to perform the task for periods of $70 \mathrm{~s}$, interleaved with rest periods of $30 \mathrm{~s}$ (see Fig. $1 \mathrm{~A}$ ). Six task plus rest periods formed a block, and subjects completed five blocks. Between blocks, subjects were given rests of $3 \mathrm{~min}$. During the voluntary contractions, magnetic stimuli (TMS; for details, see below) were applied with an intertrial interval of $5.1 \mathrm{~s}$. Despite the fact that the TMS pulses were predictive in time, we did not find significant pre-TMS trends. A total of 420 trials (TMS pulses) were obtained during an experimental session.

Electrophysiological recordings. EMG activity from the FDI muscle was recorded bipolarly. EMG signals were acquired using standard procedures $(10,000 \mathrm{~Hz})$. The impedance of EMG electrodes was below $20 \mathrm{k} \Omega$.

Electroencephalogram (EEG) was recorded from $24 \mathrm{Ag} / \mathrm{AgCl}$ electrodes placed on a subset of the 10/10 system concentrated over motor cortex, and, offline, each electrode was re-referenced to the four nearest neighbor electrodes, such that re-referenced EEG was obtained from positions $\mathrm{C} 3, \mathrm{C} 1, \mathrm{Cz}, \mathrm{C} 2, \mathrm{C} 4, \mathrm{FC} 3, \mathrm{Fc} 1, \mathrm{FCz}, \mathrm{FC} 2$, and FC4. EEG and bipolar electro-oculogram (EOG) were acquired using standard procedures $(2000 \mathrm{~Hz})$. The impedance of EEG electrodes was below $5 \mathrm{k} \Omega(20 \mathrm{k} \Omega$ for the EOG).

All signals were recorded continuously during the entire duration of the task.

Magnetic stimulation. TMS was applied using a circular coil $(90 \mathrm{~mm}$ diameter) connected to a Magstim BiStim ${ }^{2}$ stimulator (Magstim Company). The coil was positioned over the vertex of the skull with the "A-side" visible and fixated with a mechanical support. In this way, each stimulus induced a posteroanterior current flow through the left motor cortex. At the beginning of an experimental session, the active motor threshold was determined. To this end, TMS was applied while subjects maintained an ongoing voluntary contraction of the FDI at $15 \%$ of the subject's MVC. Active motor threshold was defined as the minimum stimulation intensity that elicited a motor-evoked potential (MEP) of $>200 \mu \mathrm{V}$ peak-to-peak, in at least 5 of 10 successive stimulations. Magnetic stimulation intensity during task performance was set to $110 \%$ of the subjects' individual active motor threshold. On average, the stimulus intensity used was $35 \pm 6 \%$ (mean $\pm \mathrm{SD}$ ) of maximum stimulator output $(2.0 \mathrm{~T})$.
Electrophysiological signal preprocessing. Data were analyzed offline using the FieldTrip open source MATLAB toolbox (http://fieldtrip. fcdonders.nl/; MathWorks). This included artifact rejection, powerline artifact removal, and linear detrending, yielding on average 296 artifact-free trials per subject.

The raw EMG signal was cut into epochs of $\pm 1.1 \mathrm{~s}$ around the TMS pulse. These epochs contained a small TMS artifact that was restricted to the first $1.5 \mathrm{~ms}$ ( 15 samples) after the TMS pulse. The EMG signal was bandpass filtered between 10 and $400 \mathrm{~Hz}$ (fourth-order Butterworth). Filtering was performed only forward in time, i.e., causal, to prevent any post-TMS effect from leaking into pre-TMS time. Subsequently, the preTMS EMG was demodulated to estimate the EMG amplitude. In agreement with previous literature, we will address the EMG amplitude often simply as EMG. During demodulation, the signal is Hilbert transformed, which gives the analytic signal, and then the absolute of the analytic signal is taken. This corresponds to an estimate of the time-varying total power of the EMG signal. The demodulation results in a signal that is similar to full-wave rectification of the EMG signal (Myers et al., 2003). The postTMS EMG signal was not demodulated, because it was used for determining the MEP.

Spectral analysis of prestimulus epochs. We estimated the phase of the EMG rhythm immediately preceding the TMS pulse for all frequencies between 5 and $70 \mathrm{~Hz}$, in steps of $1 \mathrm{~Hz}$. For each frequency, we used an epoch that had a length of two cycles at that frequency and that ended with the TMS pulse. This epoch was multiplied with a Hanning taper and Fourier transformed to give the phase and amplitude at the respective frequency.

Assessing the relation between pre-TMS EMG phase and post-TMS MEP amplitude. We used the frequency-wise estimate of the pre-TMS EMG phase to bin the trials. We defined 20 phase bins on the unit circle, with their centers equally spaced between $-\pi$ and $\pi$ (see Fig. $2 A$ ). To each bin, we assigned the 50 trials in which the pre-TMS EMG phases were closest to the center phase of the bin. Within each group of 50 trials, we then averaged the post-TMS EMG signal (non-demodulated) to obtain the MEP for that phase bin. The amplitude of the MEP was quantified by its peak-to-peak amplitude, i.e., the difference between the lowest and highest value within $15-50 \mathrm{~ms}$ after the TMS pulse. Also, within each group of 50 trials, we averaged (in the complex domain) the phases of the pre-TMS EMG rhythm, because this average phase per bin always differed slightly from the target phase of the respective phase bin. This procedure resulted, per subject and per frequency, in 20 pairs (one per phase bin) of pre-TMS EMG phase and post-TMS MEP amplitude (see Fig. 2C). We then (least-squares) fitted a cosine function to the MEP amplitudes as a function of the EMG phases, to quantify their dependence.

Note that, in the binning procedure, a single data epoch was typically assigned to more than one bin. For this reason, we chose subsequent statistical methods (see below) that were not affected by this partial dependence between bins. We also tried other binning parameters (more or less bins or trials per bin) and found that the results did not depend on a specific parameter set.

Estimation of additive component. To estimate a putative additive component (see Results), the following procedure was performed. The spectral analysis as described above was performed again, but, rather than being end aligned to the TMS pulse, it was now end aligned to the time point $100 \mathrm{~ms}$ before each TMS pulse. The phase binning was done accordingly, and we refer to this binning as the "control binning." We could then estimate the size of a potential additive effect. To this end, we created a template MEP waveform for each subject, by averaging all (non-demodulated) EMG signals from 0 to $0.1 \mathrm{~s}$ after TMS. This template MEP waveform was mathematically added on the (control binwise) averages of the (non-demodulated) EMG signal between $-0.1 \mathrm{~s}$ before TMS and the TMS pulse. As in the regular analysis, this procedure resulted, per subject and per frequency, in 20 pairs (one per phase bin) of EMG phase and MEP amplitude but now exclusively estimating a potential additive component. Figure $2 D$ shows the results of such an analysis in one example subject. There was no appreciable additive component in this case. To rule out any influence of a potential additive component, we subtracted (per phase bin, frequency, and subject) the estimated additive 
A

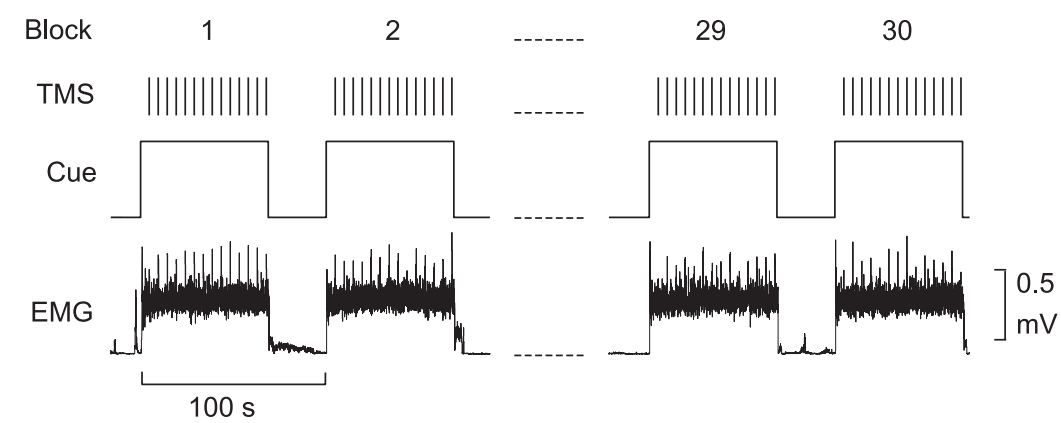

B

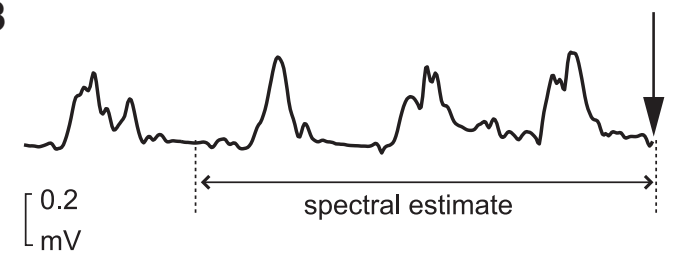

$50 \mathrm{~ms}$

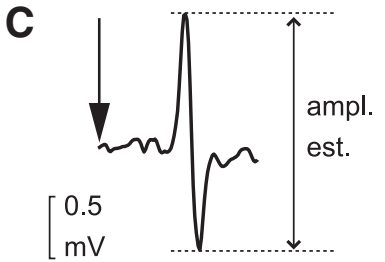

$50 \mathrm{~ms}$

Figure 1. Experimental design, example data, and task-induced rhythmic activity. $\boldsymbol{A}$, Subjects were required intermittently (70s per epoch, separated by rest periods) to produce an EMG output with their index finger, at 15\% of the amplitude measured during an earlier maximal voluntary contraction. Subjects received visual feedback about the required and the actual output level. During each epoch, 14 TMS pulses were applied, with intervals of 5.1 s between consecutive pulses. $\boldsymbol{B}$, Example pre-TMS EMG trace: rhythmic spinal motor neuron activity in the epoch just before the TMS pulse (downward arrow) was assessed by a Fourier decomposition of the EMG amplitude envelope. $\boldsymbol{C}$, The spinal response was assessed by the peak-to-peak amplitude of the TMS-evoked muscle response, the MEP.

effect throughout our analysis. This had no appreciable influence on any of the results.

Testing significance of EMG phase-dependent MEP amplitude. Figure $2 C$ shows that the relation between pre-TMS EMG phase and post-TMS MEP amplitude was cosine shaped. We therefore quantified it by (leastsquares) fitting a cosine function with the phase unconstrained (shown in Fig. $2 C$, dashed line). The modulation depth (peak-to-peak difference) of the fitted cosine was used as estimate of the strength of the relationship. For subsequent statistics, which combined cosine amplitudes across subjects, these amplitudes were normalized by the SD of the MEP amplitude estimated using a jackknife procedure (Efron and Tibshirani, 1993). The normalized amplitudes of the fitted cosines were computed for all frequencies, yielding a spectrum of normalized cosine-fit amplitudes (see Fig. $3 A$, solid line).

Cosine fits with unconstrained phases have amplitudes with a positive bias. We estimated this bias per subject by randomly shuffling pre-TMS EMG phases (independent variable) versus post-TMS MEP amplitudes (dependent variable) and repeating the above described analysis. This randomization was repeated 100 times per subject, and the average was taken as bias estimate of that subject. The dashed line in Figure $3 A$ shows the average bias estimate across subjects.

This gave two spectra per subject: one spectrum of the effect and one of the bias estimate. Our null hypothesis was that the effect spectrum was not greater than the bias spectrum and hence that the two were exchangeable. We tested this using a nonparametric randomization approach (Maris and Oostenveld, 2007). We choose this approach for several reasons. First, it is free of assumptions about the underlying distributions. Second, it is not affected by the fact that there was partial dependence (attributable to overlap) between neighboring frequency bins and also neighboring phase bins. Third, it offers an elegant way to correct for multiple comparisons. The procedure was as follows.

(1) A non-multiple comparisons corrected significance threshold was determined. (a) We defined the average difference between the effect and the bias as our test statistic. The average was taken across subjects and separately for each frequency. (b) We randomly exchanged the effect and the bias per subject. We did this for all possible permutations, given our 13 subjects, i.e., $2^{13}=8192$ times. (c) After each randomization, we determined the test statistic and entered it into a histogram, separately for

each frequency. (d) After all possible randomizations, we determined, separately for each frequency, the value of the test statistic that corresponded to the 95th percentile of this randomization distribution. This gave the nonmultiple comparisons corrected significance threshold for a one-sided test. A one-sided test was justified, because we compared against the bias and the effect should never be significantly below the bias.

(2) A cluster-based inferential statistic was performed with multiple comparisons correction. (a) For all possible permutations (see step $1 b$ ), we determined the frequency-wise test statistic. (b) We compared this test statistic against the significance threshold (from step 1d), separately for each frequency. (c) This resulted in clusters of significant adjacent frequencies for which we determined the sum of the test statistic. This sum was our cluster-level test statistic. (d) For each randomization, only the largest cluster-level test statistic across all clusters was retained and placed into a histogram. (e) After all possible randomizations, we determined the value of the cluster-level test statistic that corresponded to the 95th percentile of this randomization distribution. This gave the multiple comparisons corrected significance threshold for a one-sided test (same justification as above). (f) Steps $2 \mathrm{a}-\mathrm{c}$ were then done for the nonrandomized data, resulting in clusters with corresponding (nonrandomized) cluster-level test statistics. (g) The nonrandomized cluster-level test statistics were compared against the multiple comparison corrected significance threshold from step $2 \mathrm{e}$.

Assessing the relation between pre-TMS EMG power and post-TMS MEP amplitude. To assess for a relation between MEP amplitude and EMG power just before the TMS pulse, the preprocessed data epochs were sorted and averaged according to the spectral power of the EMG. Per channel and frequency, EMG epochs were binned according to the spectral power. We defined 20 bins, with their centers equally spaced between the minimum and maximum power values obtained for that frequency. To each bin, we assigned the 50 epochs of which the power was closest to the center power of that bin. Subsequently, the power spectra and post-TMS EMG signals were averaged within each bin. This procedure resulted, per subject and per frequency, in 20 pairs (one per power bin) of pre-TMS EMG power and post-TMS MEP amplitude. We then determined the Spearman's rank correlation coefficient between the MEP amplitudes and the EMG power values, to quantify their dependence.

Testing significance of EMG power-dependent MEP amplitude. Spearman's rank correlation coefficients were computed across all frequencies, yielding a spectrum of correlation coefficients. Although for Spearman's rank correlation coefficients no bias is expected, we nevertheless, for consistency, performed the same bias estimation procedure as had been used for the phase-dependence analysis and confirmed bias estimates close to zero (see Fig. $3 D, E$, dashed lines). Replacing the estimated bias by the expected zero bias left the outcome of statistical testing unchanged. We estimated the bias per subject by randomly shuffling preTMS EMG power (independent variable) versus post-TMS MEP amplitudes (dependent variable) and repeating the above described analysis for determining the Spearman's rank correlation coefficients. This randomization was repeated 100 times per subject, and the average was taken as bias estimate of that subject. As in the phase-dependence analysis, this gave two spectra per subject: one spectrum of the effect and one of the bias estimate. The significance testing therefore proceeded exactly as explained above for the phase-dependence analysis.

Assessing the relation between pre-TMS EEG phase or EEG power and post-TMS MEP amplitude. To test for a dependency of post-TMS MEP amplitude on the pre-TMS phase or power of the EEG, we determined 
the pre-TMS EEG phase and the pre-TMS EEG power. For all these measures, we repeated the same analyses as we had done for the EMG phase and power, with the following differences. (1) Whereas EMG data had been demodulated to estimate the EMG amplitude, this was not necessary for the EEG data. (2) Whereas there was only one differential EMG recording, there were 10 EEG channels. Only one of them is shown in Figure 3, $D$ and $E$, namely the one labeled C3, overlying contralateral motor cortex. Neither this nor any other EEG channel showed significant effects, even without correcting for multiple comparisons across the multiple channels (but correcting for the multiple comparisons across frequencies, as in all analyses).

Assessing the relation between pre-TMS EEGEMG phase relation and post-TMS MEP amplitude. To test for a dependency of post-TMS MEP amplitude on the pre-TMS phase relation between EEG and EMG, we determined the trialwise pre-TMS EEG-EMG phase relation and then repeated the analysis as for the EMG or EEG phase but with the cosine peak constrained to be at the mean EEG-EMG phase.

\section{Results}

We recorded the EMG of the first dorsal interosseus muscle while subjects $(n=13)$ held this muscle isometrically contracted (Fig. 1). During isometric contraction, the motor system engages in rhythmic synchronization in the beta-frequency range (Conway et al., 1995; Schoffelen et al., 2005, 2008). This beta-band synchronization involves also the muscle, as can be seen from the rhythmicity of the example EMG in Figure $1 B$. Muscle fibers form motor units with their innervating spinal motor neurons. EMG recordings from the muscle therefore correspond to slightly delayed recordings of multiunit activity from a functional group of spinal motor neurons. These spinal motor neurons receive synaptic input among others from motor cortex contralateral to the respective muscle. We applied TMS to contralateral motor cortex to generate precisely timed corticospinal input volleys. TMS pulses were given at random times relative to the spinal beta rhythm. This enabled us to test whether the phase of the spinal beta rhythm at which the input arrived modulated the gain of this input.

Gain is the ratio between input and output. The spinal output generated after a TMS pulse is a muscle twitch that corresponds to the MEP in the EMG recordings (Fig. $1 C$ ). We assessed the peak-to-peak amplitude of the MEP as a function of the beta phase at which the TMS pulse was applied. To this end, we sorted the trials into bins according to the beta phase immediately preceding the TMS pulse (Fig. $2 A$ ) and determined the MEP amplitude separately for each phase bin (Fig. $2 B$ ). MEP amplitude was a smooth cosine-shaped function of pre-TMS beta phase (Fig. $2 C$ ). This demonstrates that the physiological beta rhythm of the motor system entails rhythmic gain changes.

One important concern is that the observed effect might be attributable to a simple addition of the average MEP onto the beta rhythm, i.e., the observed modulation of the MEP might be fully explained by the fact that the MEP falls onto different phases of Results).

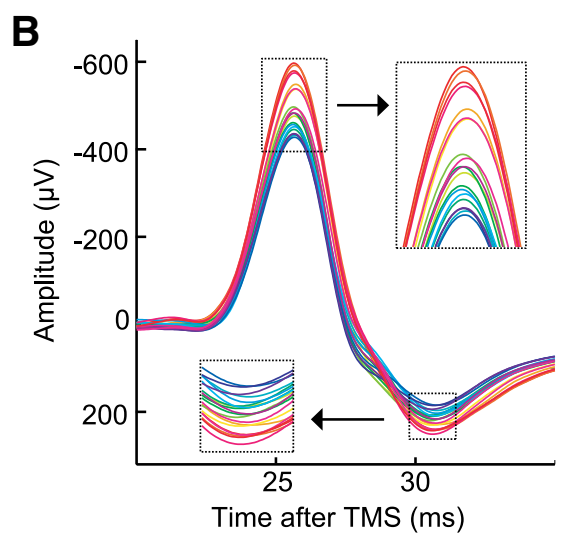

D

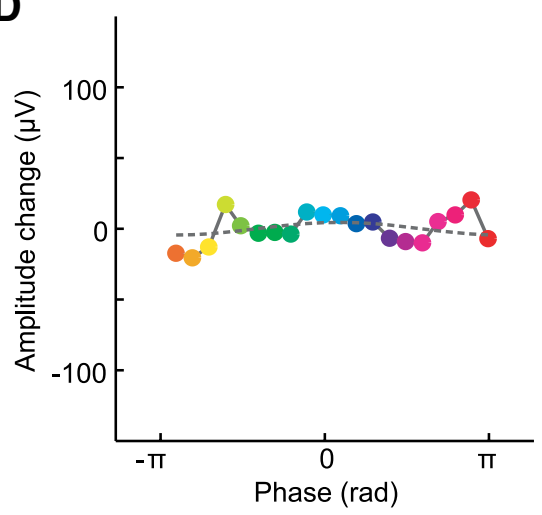

Figure 2. Pre-TMS phase determines MEP amplitude. $\boldsymbol{A}$, Gray vectors illustrate the pre-TMS phase of the EMG. Circle segments simple addition of rhythmic activity on the average MEP (same example subject; for details, see Materials and Methods and

the beta rhythm, after the phase-sorting procedure. When, during the phase-sorting procedure, we select in a given beta-phase bin e.g., the trials with an EMG trough at the TMS pulse, then those trials will have a rising EMG phase $20 \mathrm{~ms}$ later, at a typical MEP latency. Thus, sorting of trials according to pre-TMS phase leads to phase constraining some time later because of the underlying oscillation. The MEP will therefore be superimposed onto different phases of the beta rhythm for different pre-TMS phase bins. It is conceivable that this simple superposition explains the observed MEP modulation. We hypothesized, however, that the MEP modulation goes beyond such a superposition and entails a multiplicative modulation of the spinal response to the TMSinduced synaptic input to the spinal cord. The null hypothesis is that the MEP modulation can be fully explained as a superposition, and we therefore needed to estimate the MEP modulation that can be explained through superposition. For this estimation, we needed in turn estimates of the two superimposed components, i.e., the unmodulated MEP, and the phase-constrained EMG at the time of the average MEP. For an estimate of the phase-constrained EMG at the time of the average MEP, we phase constrained EMG epochs that lacked the TMS pulse (for details, see Materials and Methods). For an estimate of the unmodulated MEP, we computed the MEP across all trials. We then mathematically added this MEP onto the phase-constrained EMG epochs. This direct estimation of a potential additive component revealed that such a component, if present at all, was negligible (Fig. 2D). 

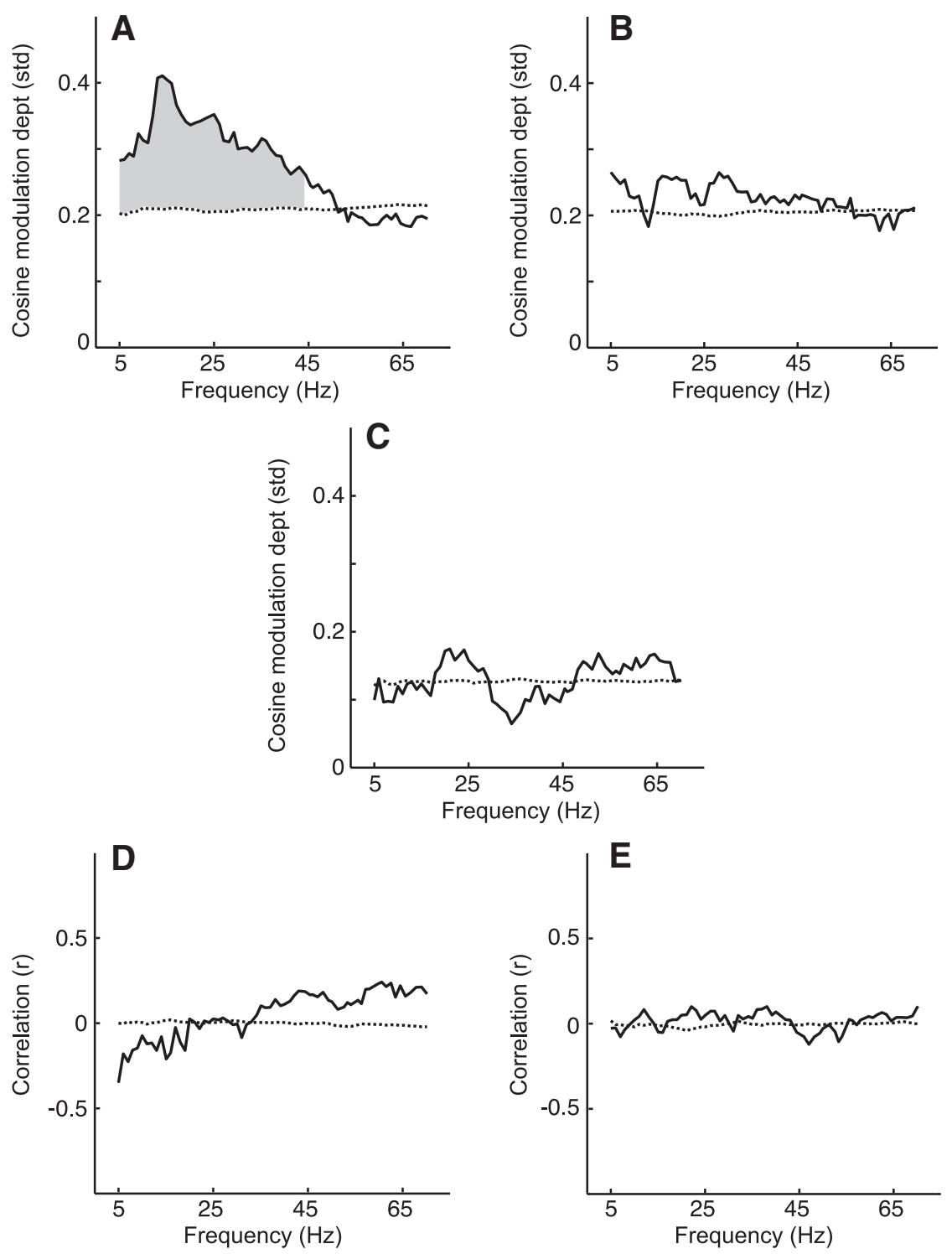

Figure 3. Group level results. $\boldsymbol{A}-\boldsymbol{E}$, Frequency spectra of MEP amplitude modulation by pre-TMS neurophysiological activity. To test for significance, the observed spectra (solid lines) were compared against their bias estimates (broken lines; see Materials and Methods). Shaded areas indicate frequency bands with significant modulations ( $n=13$ subjects; $p<0.001$, nonparametric randomization test, corrected for multiple comparisons). $\boldsymbol{A}$, Modulation of MEP by pre-TMS phase of the EMG. To combine data across subjects, the MEP modulation was normalized by the SD across trials, estimated with a jackknife procedure. $\boldsymbol{B}$, Same as $\boldsymbol{A}$ but for pre-TMS phase of the EEG recorded over the corresponding motor cortex. C, Modulation of MEP by pre-TMS phase relation between EEG and EMG. D, Spearman's correlation coefficients between MEP amplitude and pre-TMS power of the EMG. $\boldsymbol{E}$, Same as $D$ but for pre-TMS power of the EEG.

Nevertheless, all subsequent analyses were performed with and without subtracting the estimated additive component, and this had no appreciable effect on any of the results. We report the results with the additive component subtracted.

The results illustrated in Figure 2 for one example subject were found consistently across the group of 13 subjects. Per subject, we quantified the phase-dependent MEP modulation by fitting a cosine function and normalized the cosine modulation depth by the SD of the MEP across trials. This normalized cosine modulation depth was averaged across subjects and compared with a bias estimate (Fig. 3A). The entire procedure described so far for the beta rhythm was performed for a range of frequencies. Between 5 and $44 \mathrm{~Hz}$, the phase-dependent gain modulation was significant across subjects (Fig. $3 A$ ).
So far, we considered the beta rhythm in the spinal cord (as measured by EMG) and the TMS-triggered synaptic input to the spinal cord. However, the TMS pulse is applied to the motor cortex, and the spinal beta rhythm is (partially) coherent with the motor cortical beta rhythm (Conway et al., 1995; Schoffelen et al., 2005). Correspondingly, the observed effect might have its origin in the motor cortex and become visible in the spinal cord because of the (partial) corticospinal coherence. A cortical origin of the effect would be in line with our hypothesis, which is independent of the location of the effect. However, to test for a cortical origin, we had simultaneously recorded the EEG over motor cortex. The EEG reflects synchronized activity of underlying neuronal groups. We repeated the analysis but this time relating the MEP amplitude to the pre-TMS phase of the EEG. This analysis did not reveal any significant effect (Fig. 3B). We also tested whether the phase relation between motor cortex and spinal cord affected the MEP and again found no significant effect. (Fig. 3C).

Finally, we considered that the observed effect might be attributable to a confounding role of EMG power. Variance in EMG power can typically explain part of the variance in MEP amplitude (Hess et al., 1987). The observed relation between MEP amplitude and pre-TMS EMG phase might therefore be confounded by a potential relation between pre-TMS EMG phase and pre-TMS EMG power. We therefore tested whether preTMS EMG power predicted MEP amplitudes and found no relation (Fig. 3D). The absence of a relation between EMG power and MEP amplitude in our data is likely attributable to the fact that visual online feedback of the EMG resulted in a very small EMG power variance. We tested also whether MEP amplitude is related to the pre-TMS power of EEG, and this analysis did not reveal any significant relation (Fig. 3E).

Having established that the spinal beta rhythm entails a rhythmic gain modulation, we asked whether the phase-gain relationship was physiologically plausible. To this end, we selected for each subject the beta-rhythm phase bin that resulted in maximal gain and investigated the EMG from the trials in that bin (Fig. $4 A, B)$. This analysis demonstrated that, across subjects, TMS pulses resulting in maximal MEPs were preceded by a specific beta-rhythm phase. This phase was obtained at the EMG level while the TMS pulse was delivered at the cortical level. To estimate the corresponding, optimal, phase at the spinal cord level, we could simply extrapolate the phase obtained at the EMG level to the latency of the MEP (Fig. $4 B$, gray cosine). The MEP results from a spinal output volley that travels to the muscle at the same speed as the ongoing beta-rhythmic volleys. Therefore, the 
extrapolated EMG phase at MEP onset corresponds to the spinal phase with maximal input gain. The green vertical line in Figure $4 B$ indicates the average MEP latency, and the cosine fit demonstrates that maximal spinal input gain occurred around the moment of steepest rise in the ongoing beta rhythm. Figure $4, C$ and $D$, illustrates that minimal spinal input gain occurred around the moment of steepest decline in the ongoing beta rhythm.

\section{Discussion}

In summary, we find that an ongoing beta rhythm of a neuronal group systematically modulates the response of that group to input. Crucial aspects of this result are as follows.

(1) The investigated rhythm occurs in vivo, as a physiological rhythm involved in long-range interaction in the motor system, and it is intrinsically generated, i.e., not imposed through an external stimulus.

(2) The rhythm is at a relatively high frequency, the beta band. Beta-band synchronization has been implicated in longrange interactions among brain areas by numerous studies (Tallon-Baudry et al., 2001, 2004; Brovelli et al., 2004; Gross et al., 2004; Buschman and Miller, 2007). For other effector muscles and movement conditions, the frequency can be higher or lower (Brown et al., 1998; McAuley and Marsden, 2000; Wolpaw and McFarland, 2004; Schoffelen et al., 2005; Mellinger et al., 2007), and it will be an interesting topic for future research to test whether the effect described here holds for those other frequency bands.

(3) The phase-dependent response modulation was not attributable to a linear summation of the ongoing rhythm onto an unmodulated response. An estimate of a contribution of linear summation effects revealed that they were completely absent. Rather, we observed an interaction of the phase of the ongoing rhythm with the input that constituted a modulation of multiplicative input gain. A related effect has been described in monkey auditory cortex (Lakatos et al., 2007). There, somatosensory stimulation leads to an evoked response that is roughly opposite in phase for contralateral versus ipsilateral stimulus location. The response to a simultaneous auditory stimulus is enhanced by contralateral somatosensory stimulation and vice versa.

(4) The phase-dependent response was well approximated by a cosine function. This suggests that the (multiplicative) gain depends linearly on phase, in agreement with a recent study indicating that interaction strength depends linearly on phase relation (Womelsdorf et al., 2007).

(5) The phase-dependent effect was not confounded by an amplitude-dependent effect, because EMG amplitude did not predict MEP size.

(6) We could determine the actual phase that resulted in maximal input gain. We found that synaptic input to the spinal cord is most effective when it arrives in the rising phase of the ongoing spinal beta rhythm. This corresponds to maximal MEPs elicited by TMS pulses delivered close to the trough of the EMG beta

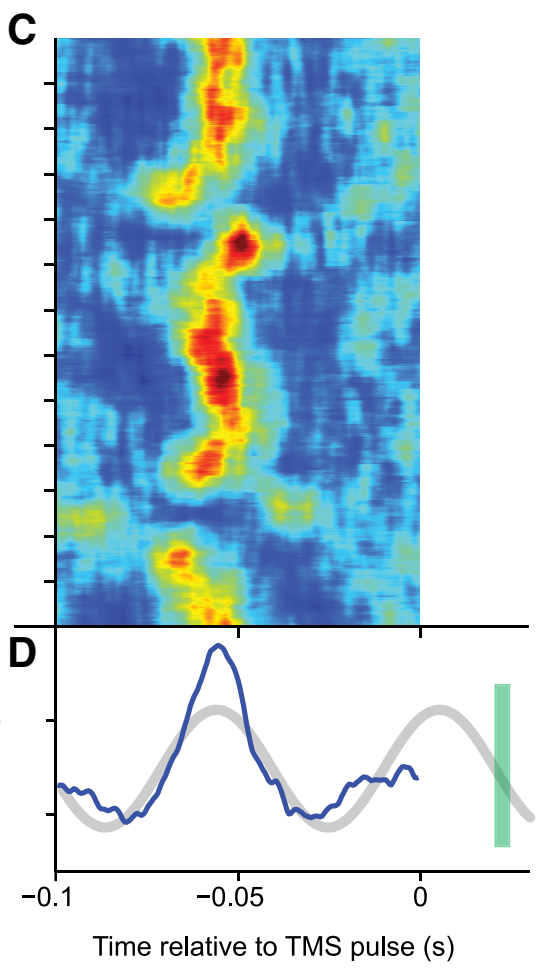

Figure 4. Synaptic input is most effective when arriving at rising phase of spinal beta rhythm. A, Pre-TMS epochs of EMG 政

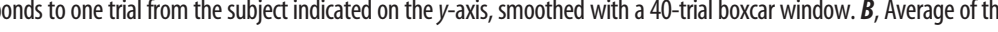
(1)

rhythm. This timing, close to the trough of the EMG is actually found for spikes of pyramidal tract neurons during physiological beta-band coherence (Baker et al., 1997). Thus, physiological beta-band coherence leads to spikes optimally timed for impact onto the spinal cord. Related analyses of ongoing or evoked phases that lead to enhancement or suppression of neuronal responses have been performed previously in other systems and frequency bands (Kruglikov and Schiff, 2003; Lakatos et al., 2007, 2008; Rajkai et al., 2008).

We tested whether the MEP depends also on the phase of the EEG over motor cortex and did not find a significant effect there. The absence of a significant cortical effect might appear surprising, because the cortex is coherent with the spinal cord. However, this coherence is relatively weak, with coherence values that are typically (and also in our dataset) around 0.1 . This relatively weak coherence might explain why the effect that reaches significance for the spinal phase does not reach significance for the cortical phase. In the cortex, a similar gain modulation effect becomes visible only when the gain of synaptic input in cortex is investigated (Kruglikov and Schiff, 2003; Lakatos et al., 2007, 2008; Rajkai et al., 2008). In contrast, the current analysis probed whether the cortical phase modulates the impact of TMS, which is conveyed to a large degree through direct electromagnetic stimulation of the corticospinal cells and their axon initial segments. Together, the significant spinal and nonsignificant cortical effects are most parsimoniously explained by a spinal origin of the effect.

Several previous studies have related stimulus (or generally input)-driven neuronal responses to ongoing rhythmic neuronal activity. Briggs and Usrey (2007) demonstrated that visual cortical neurons are more likely to respond to electrical LGN stimu- 
lation when their activity had been elevated 30-40 ms before stimulation. This suggests that the phase of gamma activity might contribute to geniculocortical communication. Recently, Cardin et al. (2009) used optogenetic techniques to impose a gammafrequency rhythm on barrel cortex of anesthetized rats and demonstrated that this imposed rhythm modulated the response to whisker stimulation.

Rajkai et al. (2008) and Lakatos et al. (2005, 2008) demonstrated that the sensory-driven response of awake monkey visual or auditory cortex depends on the phase of the preceding ongoing rhythm, which was either spontaneously present or imposed by an approximately rhythmic sensory stimulation. The respective rhythms were either a spontaneous 3-8 Hz rhythm (Rajkai et al., 2008) or they were stimulus-entrained 1-2 Hz rhythms (Lakatos et al., 2005, 2008). Another study by Lakatos et al. (2007) demonstrated that somatosensory stimulation leads to an evoked response in auditory cortex that is roughly opposite in phase for contralateral versus ipsilateral somatosensory stimulation. The response to a simultaneous auditory stimulus is enhanced by contralateral somatosensory stimulation and vice versa. Finally, Kruglikov and Schiff (2003) triggered auditory stimuli with different delays after troughs in the EEG and reported a delaydependent response modulation.

One recent study related pre-TMS EMG oscillations to the size of the MEP in a different context (Mitchell et al., 2007). The MEP size varies considerably from trial to trial, and these authors therefore aimed at explaining as much of this variability as possible. To this end, they modeled the MEP as a function of both the phase and amplitude of pre-TMS EMG combined, and they found a significant fraction of variance explained like this. Although the combined consideration of phase and amplitude was optimal to explain variance, our motivation required to isolate phase from amplitude and to actually determine the phase of maximal input gain.

The present study shows that, for a physiological in vivo rhythm, the response to a single short-lasting input depends on the phase of the rhythm of the target at which the input arrives. Similar evidence had been obtained previously from in vitro brain slice preparations. Burchell et al. (1998) used glutamate ejection onto hippocampal slices to produce population spikes at a $24-42$ $\mathrm{Hz}$ rhythm. The involved neurons responded to electrically evoked Schaffer collateral inputs with a gain that depended systematically on the delay from the last population spike. This phase-dependent input gain is most likely attributable to the rhythmic inhibition after population spikes and might be related to what we describe in vivo, namely maximal gain for the rising phase of the rhythmic activity of the target.

The results presented here demonstrate that the gain of input is modulated by the phase of the rhythm of the neuronal target group. This result might have been expected based on the rhythmic inhibition involved in local beta/gamma-band synchronization (Kopell et al., 2000). However, it was crucial to establish it in vivo, because it might be a fundamental mechanism underlying flexible neuronal communication. The flexible modulation of neuronal communication is at the heart of cognition, and several mechanisms have been proposed (Salinas and Thier, 2000). We put forward a mechanism with an important advantage: in many cases in which gain modulation is considered crucial, it is supposed to act on a neuronal connection rather than on a neuronal source or target group (Reynolds et al., 1999). Neuronal connections could in principle be modulated by affecting (groups of) synapses, but such mechanisms require to actually target the appropriate sets of synapses, which would require the flexible rout- ing of neuromodulatory inputs to changing subsets of synapses. In contrast, the mechanism proposed here requires merely the synchronization between the selected source and target group but still implements a gain modulation selectively for the synchronized neuronal connection.

Most mechanisms proposed so far for gain modulation modulate the gain for the entire duration of the cognitive episode that requires the corresponding gain change. In contrast, we propose that the target group modulates input gain rhythmically and thereby multiplexes input gain along the phases of the cycle of its rhythm. The actual gain of an input is then determined by the phase and precision of the synchronization between input and target.

Finally, we note that high-frequency synchronization is often modulated by the phase of low-frequency rhythms (Bragin et al., 1995; Lakatos et al., 2005; Canolty et al., 2006; Bosman et al., 2009; Fries, 2009a; Schroeder and Lakatos, 2009). Low-frequency rhythms even appear to switch between alternative spatial gamma-synchronization patterns (Colgin et al., 2009; Fries, 2009b). Thus, low-frequency rhythms might modulate neuronal communication both directly, by slowly modulating neuronal excitability, and indirectly, by rhythmically modulating the strength and the spatial pattern of higher-frequency synchronization. The relative roles of low- and high-frequency synchronization and their interplay are important targets for future research.

\section{References}

Baker SN, Olivier E, Lemon RN (1997) Coherent oscillations in monkey motor cortex and hand muscle EMG show task-dependent modulation. J Physiol 501:225-241.

Börgers C, Kopell NJ (2008) Gamma oscillations and stimulus selection. Neural Comput 20:383-414.

Börgers C, Epstein S, Kopell NJ (2005) Background gamma rhythmicity and attention in cortical local circuits: a computational study. Proc Natl Acad Sci U S A 102:7002-7007.

Bosman CA, Womelsdorf T, Desimone R, Fries P (2009) A microsaccadic rhythm modulates gamma-band synchronization and behavior. J Neurosci 29:9471-9480.

Bragin A, Jandó G, Nádasdy Z, Hetke J, Wise K, Buzsáki G (1995) Gamma $(40-100 \mathrm{~Hz})$ oscillation in the hippocampus of the behaving rat. J Neurosci 15:47-60.

Briggs F, Usrey WM (2007) Cortical activity influences geniculocortical spike efficacy in the macaque monkey. Front Integr Neurosci 1:3.

Brovelli A, Ding M, Ledberg A, Chen Y, Nakamura R, Bressler SL (2004) Beta oscillations in a large-scale sensorimotor cortical network: directional influences revealed by Granger causality. Proc Natl Acad Sci U S A 101:9849-9854.

Brown P, Salenius S, Rothwell JC, Hari R (1998) Cortical correlate of the Piper rhythm in humans. J Neurophysiol 80:2911-2917.

Burchell TR, Faulkner HJ, Whittington MA (1998) Gamma frequency oscillations gate temporally coded afferent inputs in the rat hippocampal slice. Neurosci Lett 255:151-154.

Buschman TJ, Miller EK (2007) Top-down versus bottom-up control of attention in the prefrontal and posterior parietal cortices. Science 315:1860-1862.

Canolty RT, Edwards E, Dalal SS, Soltani M, Nagarajan SS, Kirsch HE, Berger MS, Barbaro NM, Knight RT (2006) High gamma power is phaselocked to theta oscillations in human neocortex. Science 313:1626-1628.

Cardin JA, Carlén M, Meletis K, Knoblich U, Zhang F, Deisseroth K, Tsai LH, Moore CI (2009) Driving fast-spiking cells induces gamma rhythm and controls sensory responses. Nature 459:663-667.

Colgin LL, Denninger T, Fyhn M, Hafting T, Bonnevie T, Jensen O, Moser MB, Moser EI (2009) Frequency of gamma oscillations routes flow of information in the hippocampus. Nature 462:353-357.

Conway BA, Halliday DM, Farmer SF, Shahani U, Maas P, Weir AI, Rosenberg JR (1995) Synchronization between motor cortex and spinal motoneuronal pool during the performance of a maintained motor task in man. J Physiol 489:917-924. 
Csicsvari J, Jamieson B, Wise KD, Buzsáki G (2003) Mechanisms of gamma oscillations in the hippocampus of the behaving rat. Neuron 37:311-322.

Efron B, Tibshirani RJ (1993) An introduction to the bootstrap. Boca Raton, FL: Chapman and Hall/CRC.

Fries P (2005) A mechanism for cognitive dynamics: neuronal communication through neuronal coherence. Trends Cogn Sci 9:474-480.

Fries P (2009a) Neuronal gamma-band synchronization as a fundamental process in cortical computation. Annu Rev Neurosci 32:209-224.

Fries P (2009b) The model- and the data-gamma. Neuron 64:601-602.

Fries P, Nikolić D, Singer W (2007) The gamma cycle. Trends Neurosci 30:309-316.

Gross J, Schmitz F, Schnitzler I, Kessler K, Shapiro K, Hommel B, Schnitzler A (2004) Modulation of long-range neural synchrony reflects temporal limitations of visual attention in humans. Proc Natl Acad Sci U S A 101:13050-13055.

Hasenstaub A, Shu Y, Haider B, Kraushaar U, Duque A, McCormick DA (2005) Inhibitory postsynaptic potentials carry synchronized frequency information in active cortical networks. Neuron 47:423-435.

Hess CW, Mills KR, Murray NM (1987) Responses in small hand muscles from magnetic stimulation of the human brain. J Physiol 388:397-419.

Kopell N, Ermentrout GB, Whittington MA, Traub RD (2000) Gamma rhythms and beta rhythms have different synchronization properties. Proc Natl Acad Sci U S A 97:1867-1872.

Kruglikov SY, Schiff SJ (2003) Interplay of electroencephalogram phase and auditory-evoked neural activity. J Neurosci 23:10122-10127.

Lakatos P, Shah AS, Knuth KH, Ulbert I, Karmos G, Schroeder CE (2005) An oscillatory hierarchy controlling neuronal excitability and stimulus processing in the auditory cortex. J Neurophysiol 94:1904-1911.

Lakatos P, Chen CM, O'Connell MN, Mills A, Schroeder CE (2007) Neuronal oscillations and multisensory interaction in primary auditory cortex. Neuron 53:279-292.

Lakatos P, Karmos G, Mehta AD, Ulbert I, Schroeder CE (2008) Entrainment of neuronal oscillations as a mechanism of attentional selection. Science 320:110-113.

Maris E, Oostenveld R (2007) Nonparametric statistical testing of EEG- and MEG-data. J Neurosci Methods 164:177-190.

McAuley JH, Marsden CD (2000) Physiological and pathological tremors and rhythmic central motor control. Brain 123:1545-1567.

Mellinger J, Schalk G, Braun C, Preissl H, Rosenstiel W, Birbaumer N, Kübler A (2007) An MEG-based brain-computer interface (BCI). Neuroimage 36:581-593.

Mitchell WK, Baker MR, Baker SN (2007) Muscle responses to transcranial stimulation in man depend on background oscillatory activity. J Physiol 583:567-579.
Murthy VN, Fetz EE (1996) Oscillatory activity in sensorimotor cortex of awake monkeys: synchronization of local field potentials and relation to behavior. J Neurophysiol 76:3949-3967.

Myers LJ, Lowery M, O’Malley M, Vaughan CL, Heneghan C, St Clair Gibson A, Harley YX, Sreenivasan R (2003) Rectification and non-linear preprocessing of EMG signals for cortico-muscular analysis. J Neurosci Methods 124:157-165.

Oldfield RC (1971) The assessment and analysis of handedness: the Edinburgh inventory. Neuropsychologia 9:97-113.

Rajkai C, Lakatos P, Chen CM, Pincze Z, Karmos G, Schroeder CE (2008) Transient cortical excitation at the onset of visual fixation. Cereb Cortex 18:200-209.

Reynolds JH, Chelazzi L, Desimone R (1999) Competitive mechanisms subserve attention in macaque areas V2 and V4. J Neurosci 19:1736-1753.

Salinas E, Thier P (2000) Gain modulation: a major computational principle of the central nervous system. Neuron 27:15-21.

Schnitzler A, Gross J (2005) Normal and pathological oscillatory communication in the brain. Nat Rev Neurosci 6:285-296.

Schoffelen JM, Oostenveld R, Fries P (2005) Neuronal coherence as a mechanism of effective corticospinal interaction. Science 308:111-113.

Schoffelen JM, Oostenveld R, Fries P (2008) Imaging the human motor system's beta-band synchronization during isometric contraction. Neuroimage 41:437-447.

Schroeder CE, Lakatos P (2009) Low-frequency neuronal oscillations as instruments of sensory selection. Trends Neurosci 32:9-18.

Singer W, Gray CM (1995) Visual feature integration and the temporal correlation hypothesis. Annu Rev Neurosci 18:555-586.

Tallon-Baudry C, Bertrand O, Fischer C (2001) Oscillatory synchrony between human extrastriate areas during visual short-term memory maintenance. J Neurosci 21:RC177(1-5).

Tallon-Baudry C, Mandon S, Freiwald WA, Kreiter AK (2004) Oscillatory synchrony in the monkey temporal lobe correlates with performance in a visual short-term memory task. Cereb Cortex 14:713-720.

Whittington MA, Traub RD, Kopell N, Ermentrout B, Buhl EH (2000) Inhibition-based rhythms: experimental and mathematical observations on network dynamics. Int J Psychophysiol 38:315-336.

Wolpaw JR, McFarland DJ (2004) Control of a two-dimensional movement signal by a noninvasive brain-computer interface in humans. Proc Natl Acad Sci U S A 101:17849-17854.

Womelsdorf T, Schoffelen JM, Oostenveld R, Singer W, Desimone R, Engel AK, Fries P (2007) Modulation of neuronal interactions through neuronal synchronization. Science 316:1609-1612. 